\title{
MINIMAL SURFACES WITH LOW INDEX IN THE THREE-DIMENSIONAL SPHERE
}

\author{
FRANCISCO URBANO
}

(Communicated by Jonathan M. Rosenberg)

\begin{abstract}
In the present paper, the author gives a characterization of the Clifford torus among the minimal surfaces of the three-dimensional sphere in terms of its index.
\end{abstract}

\section{INTRODUCTION}

Let $M$ be a compact orientable minimal surface in the three-dimensional unit sphere $S^{3}(1)$. The Jacobi operator of the second variation is given by $L=\Delta+|\sigma|^{2}+2$, where $\sigma$ is the second fundamental form of $M$ and $\Delta$ is the Laplacian of the induced metric. The index of $M, \operatorname{ind}(M)$, is defined as the number of negative eigenvalues of $L$. We remark that the stability of $M$ is equivalent to $\operatorname{Ind}(M)=0$.

In [S], J. Simons proved that $M$ cannot be stable $(\operatorname{Ind}(M) \geq 1)$ and characterized the totally geodesic immersion as the only one with index one.

In this paper, we get a lower bound of $\operatorname{Ind}(M)$ when $M$ is not totally geodesic and characterize the Clifford torus as the only compact minimal surface whose index is that lower bound. In fact, we prove the following:

Theorem. Let $M$ be a compact orientable nontotally geodesic minimal surface in $S^{3}(1)$. Then ind $(M) \geq 5$, and the equality holds if and only if $M$ is the Clifford torus.

Remark. The above theorem is also true when $M$ is a complete orientable minimal surface of $S^{3}(1)$, because in $S^{3}(1)$ any complete orientable minimal surface with finite index is compact. (See [FC] and [L-R], Theorem 4).

\section{Preliminaries}

Let $\Phi: M \rightarrow S^{3}(1)$ be an isometric immersion of a compact orientable minimal surface in $S^{3}(1)$. We denote by $\langle$,$\rangle the metric of \mathbb{R}^{4}$ as well as that

Received by the editors April 3, 1989 and, in revised form, July 28, 1989.

1980 Mathematics Subject Classification (1985 Revision). Primary 53C40.

Key words and phrases. Minimal surface, index, Jacobi operator.

Research partially supported by DGICYT grant PS87-0115-C03-02. 
induced on $S^{3}(1)$ and $M$. If $\sigma$ is the second fundamental form of $\Phi$ and $\Delta$ the Laplacian of the metric induced on $M$, then $L=\Delta+|\sigma|^{2}+2$ is the Jacobi operator of the second variation, and its associated quadratic form is given by

$$
Q(u, u)=\int_{M}\left\{|\nabla u|^{2}-\left(|\sigma|^{2}+2\right) u^{2}\right\} d A,
$$

where $d A$ is the measure of the metric on $M$. Then, $\operatorname{ind}(M)$ is the index of $Q$. If $\lambda_{i}, i=1,2, \ldots$, are the eigenvalues of $L$, and $f_{i}$ the corresponding eigenfunctions, then

$$
\lambda_{k} \leq Q(u, u)
$$

for any function $u$ with $\int_{M} u^{2} d A=1$ and $\int_{M} u f_{i} d A=0$ for $i=1,2, \ldots, k-$ 1 , and the equality in (1) holds if and only if $L u+\lambda_{k} u=0$.

Finally (see [M-R] for details), if $\mathbb{D}^{4}$ is the open unit ball in $\mathbb{R}^{4}$, then for each $g \in \mathbb{D}^{4}$ we define a conformal transformation $F_{g}$ of $S^{3}(1)$ by

$$
F_{g}(p)=\frac{p+(\mu\langle p, g\rangle+\lambda) g}{\lambda(1+\langle p, g\rangle)}
$$

where $\lambda=\left(1-|g|^{2}\right)^{-(1 / 2)}, \mu=(\lambda-1)|g|^{-2}$.

If $\Phi: M \rightarrow S^{3}(1)$ is a minimal immersion, then

$$
2 A=\int_{M}\left|\nabla\left(F_{g} \circ \Phi\right)\right|^{2} d A+2 \int_{M}\left(\frac{\langle N, g\rangle}{(1+\langle\Phi, g\rangle)}\right)^{2} d A,
$$

where $A$ is the area of $M$, and $N$ is a unit normal vector field to $M$.

\section{Proof of the theorem}

For any vector $a \in \mathbb{R}^{4}$ let $f_{a}=\langle N, a\rangle$. Then it is easy to see that $L f_{a}-2 f_{a}=$ 0 . So, -2 is an eigenvalue of $L$, and the functions on $V=\left\{f_{a} / a \in \mathbb{R}^{4}\right\}$ are eigenfunctions of -2 .

It is clear that $\operatorname{dim} V \leq 4$. If $\operatorname{dim} V \leq 3$, then there exists a nonzero vector $a$ in $\mathbb{R}^{4}$ such that $f_{a}=0$. Then a simple computation proves that the Hessian of the function $g=\langle\Phi, a\rangle$ satisfies Hess $g=-g\langle$,$\rangle , and so using Obata's$ theorem [O], we have that $M$ is either isometric to a unit sphere or $g=0$. In the first case, $M$ is totally geodesic. So $g$ must be zero. But in this case, as $a \neq 0, \Phi(M)$ lies in an equator of $S^{3}(1)$, and again $M$ is totally geodesic. So $\operatorname{dim} V=4$. Then -2 cannot be the first eigenvalue of $L$, because the multiplicity of $\lambda_{1}$ is one. So $\operatorname{ind}(M) \geq 1+\operatorname{dim} V=5$. Now suppose that $\operatorname{ind}(M)=5$. Then $\lambda_{2}=-2$. Let $\rho$ be the eigenfunction of $\lambda_{1}$ which can be taken positive. Then (see [L-Y]), there exists a conformal transformation $F_{g}$ of $S^{3}(1)$ such that

$$
\int_{M} \rho\left(F_{g} \circ \Phi\right) d A=0
$$


So from (1), we have that the components of $F_{g} \circ \Phi$ satisfy

$$
Q\left(\left(F_{g} \circ \Phi\right)_{i},\left(F_{g} \circ \Phi\right)_{i}\right) \geq-2 \int_{M}\left(F_{g} \circ \Phi\right)_{i}^{2} d A
$$

for $i=1,2,3,4$. So from (3) we have

$$
\int_{M}\left|\nabla\left(F_{g} \circ \Phi\right)\right|^{2} d A \geq \int_{M}|\sigma|^{2} d A
$$

Then using (2), we get

$$
2 A \geq \int_{M}|\sigma|^{2} d A,
$$

and the equality holds if and only if the equality holds in (3) and $\langle N, g\rangle=0$.

From Gauss's formula, we have $|\sigma|^{2}=2-2 K$, where $K$ is the Gauss curvature of $M$. So using the Gauss-Bonnet theorem, (4) implies that the genus of $M$ is less than or equal to one. If the genus is zero, using a result of Almgren [A], we have that $M$ is totally geodesic, which contradicts the assumptions. So $M$ must be a torus, and in this case the equality in (4) holds. So $\langle N, g\rangle=0$. If $f=\langle\Phi, g\rangle$, then Hess $f=-f\langle$,$\rangle , and using a similar reasoning as above,$ we get that $g=0$, and then $F_{g} \circ \Phi=\Phi$.

Now as the equality in (3) holds, too, we have that the components $\Phi_{i}$ of $\Phi$ are eigenfunctions of -2 , which implies that $\Delta \Phi_{i}=-|\sigma|^{2} \Phi_{i}$. But it is easy to see that $\Delta \Phi_{i}=-2 \Phi_{i}$. So $\left(|\sigma|^{2}-2\right) \Phi_{i}=0$ for $i=1,2,3,4$. Then $|\sigma|^{2}=2$, and using a very well-known result (see [Ch-DoC-K]), we obtain that $M$ is the Clifford torus.

Finally, if $M$ is the Clifford torus, we have that $|\sigma|^{2}=2$ and so $L=\Delta+4$. If $\mu_{i}$ are the eigenvalues of $\Delta$, then $\lambda_{i}=\mu_{i}-4$. As $\mu_{1}=0, \mu_{2}=2$ (with multiplicity 4), and $\mu_{3}=4$, we have that $\operatorname{Ind}(M)=5$.

Remark. The referee pointed out to me that the same theorem was observed independently by Doris Fischer-Colbrie, who apparently lectured on it but never published it.

\section{REFERENCES}

[A] F. J. Almgren, Jr., Some interior regularity theorems for minimal surfaces and an extension of Berstein's theorem, Ann. of Math. 84 (1966), 277-292.

[Ch-DoC-K] S. S. Chern, M. DoCarmo, and S. Kobayashi, Minimal submanifolds of a sphere with second fundamental form of constant length, Functional Analysis and Related Fields, Proc. Conf. M. Stone, Springer, 1970, pp. 59-75.

[FC] D. Fischer-Colbrie, On complete minimal surfaces with finite Morse index in three manifolds, Invent. Math. 82 (1985), 121-132.

[L-Y] P. Li and S. T. Yau, A new conformal invariant and its applications to the Willmore conjecture and first eigenvalue of compact surfaces, Invent. Math. 69 (1982), 269-291.

[L-R] F. J. Lopez and A. Ros, Complete minimal surfaces with index one and stable constant mean curvature surfaces, Comment. Math. Helvetici 64 (1989), 34-43. 
[M-R] S. Montiel and A. Ros, Minimal immersions of surfaces by the first eigenfunctions and conformal area, Invent. Math. 83 (1986), 153-166.

[O] M. Obata, Certain conditions for a Riemannian manifold to be isometric with a sphere, J. Math. Soc. Japan 14 (1962), 333-340.

[S] J. Simons, Minimal varieties in Riemannian manifolds, Ann. of Math. 88 (1968), 62-105.

Departmento de Geometria y Topologia, Universidad de Granada, 18071 Granada, SPAIN 